\title{
LOGARITHMIC CONVEXITY OF PERRON-FROBENIUS EIGENVECTORS OF POSITIVE MATRICES
}

\author{
SIDDHARTHA SAHI \\ (Communicated by Louis J. Ratliff, Jr.)
}

\begin{abstract}
Let $\mathrm{C}(S)$ be the cone of Perron-Frobenius eigenvectors of stochastic matrices that dominate a fixed substochastic matrix $S$. For each $0 \leq \alpha \leq 1$, it is shown that if $u$ and $v$ are in $\mathrm{C}(S)$ then so is $w$, where $w_{j}=u_{j}^{\alpha} v_{j}^{1-\alpha}$.
\end{abstract}

The basic result of Perron-Frobenius theory $[\mathrm{S}]$ is that if a matrix has strictly positive entries, then its maximal eigenvalue is unique, positive, occurs with multiplicity 1 , and has a (coordinatewise) positive eigenvector.

Subsequent literature on positive matrices contains many results (e.g., [C, F, $\mathrm{K}]$ ) that deal with convexity properties of the dominant eigenvalue as a function of matrix entries. Similar results for the corresponding eigenvectors are obtained in [DN, EJN] but only for the effects of varying a single row of the matrix. Little seems to be known about the behavior of these eigenvectors under a more general perturbation of the matrix.

In this paper we prove a different kind of convexity property for PerronFrobenius eigenvectors that was motivated by economic considerations in [SY] but that, by virtue of its unexpected and elementary nature, seems to warrant a wider mathematical audience.

For convenience, we formulate the result in terms of stochastic matrices-a positive matrix is called stochastic (substochastic) if its column sums are equal to (less than) 1.

For a fixed substochastic matrix $S$, consider the cone $\mathrm{C}(S)$ of all (positive) Perron-Frobenius eigenvectors of the various stochastic matrices that (entrywise) dominate $S$. Thus $\mathrm{C}(S)=\{v>0 \mid \exists$ stochastic $A \geq S$ such that $A v=$ $v\}$.

Now $\mathrm{C}(S)$ need not be a convex subset of $\mathbf{R}^{n}$. However, we shall show that it has the following remarkable property that may be termed logarithmic, or geometric, convexity.

Theorem. Fix $0 \leq \alpha \leq 1$, and put $\beta=1-\alpha$. If $u=\left(u_{1}, u_{2}, \ldots, u_{n}\right)$ and $v=\left(v_{1}, v_{2}, \ldots, v_{n}\right)$ are in $\mathrm{C}(S)$ then so is $w=\left(w_{1}, w_{2}, \ldots, w_{n}\right)$ where $w_{j}=u_{j}^{\alpha} v_{j}^{\beta}$.

Received by the editors November 13,1991 and, in revised form, December 13, 1991.

1991 Mathematics Subject Classification. Primary 15A48, 15A51.

Key words and phrases. Positive matrix, stochastic matrix, Perron-Frobenius eigenvector, convexity. 
The principal difficulty in proving this theorem is the indirect nature of the definition of $\mathrm{C}(S)$. The following lemma "eliminates the quantifier" in that definition.

Lemma. A positive vector $v$ belongs to $\mathrm{C}(S)$ if and only if $S v \leq v$.

Proof. If $v$ is in $\mathrm{C}(S)$, choose $A \geq S$ such that $v=A v$. Then clearly $S v \leq A v=v$.

Conversely, suppose $v>0$ with $S v \leq v$, and put $\delta=v-S v$. Also, let $s_{j}$ be the $j$ th column sum of $S$, and put $\varepsilon_{j}=1-s_{j}$. Clearly, $0<$ $\varepsilon_{1} v_{1}+\cdots+\varepsilon_{n} v_{n}=\delta_{1}+\cdots+\delta_{n}=\lambda$, say. Now let $A$ be the matrix whose $i j$ th entry is $a_{i j}=s_{i j}+\frac{1}{\lambda} \delta_{i} \varepsilon_{j}$. It is easily checked that $A$ is stochastic, dominates $S$, and satisfies $A v=v$.

Proof of Theorem. In view of the lemma, we may assume that $S u \leq u$ and $S v \leq v$, and we have to show that $S w \leq w$. Using the Hölder inequality, we get

$$
\begin{aligned}
(S w)_{i} & =\sum_{j} s_{i j} w_{j}=\sum_{j} s_{i j} u_{j}^{\alpha} v_{j}^{\beta}=\sum_{j}\left(s_{i j} u_{j}\right)^{\alpha}\left(s_{i j} v_{j}\right)^{\beta} \\
& \leq\left(\sum_{j} s_{i j} u_{j}\right)^{\alpha}\left(\sum_{j} s_{i j} v_{j}\right)^{\beta} \leq\left(u_{i}\right)^{\alpha}\left(v_{i}\right)^{\beta}=w_{i} .
\end{aligned}
$$

\section{REFERENCES}

[C] J. Cohen, Convexity of the dominant eigenvalue of an essentially nonnegative matrix, Proc. Amer. Math. Soc. 81 (1981), 657-658.

[DN] E. Deutsch and M. Neumann, On the first and second order derivatives of the Perron vector, Linear Algebra Appl. 71 (1985), 57-76.

[EJN] L. Elsner, C. Johnson, and M. Neumann, On the effect of the perturbation of a nonnegative matrix on its Perron eigenvector, Czech. Math. J. 32 (1982), 99-109.

[F] S. Friedland, Convex spectral functions, Linear and Multilinear Algebra 9 (1981), 299-316.

[K] J. Kingman, A convexity property of positive matrices, Quart. J. Math. 81 (1961), 283-284.

[S] E. Seneta, Non-negative matrices, George Allen \& Unwin, London, 1973.

[SY] S. Sahi and S. Yao, The non-cooperative equilibria of a trading economy with complete markets and consistent prices, J. Math. Econ. 18 (1989), 325-346.

Department of Mathematics, Princeton University, Princeton, New Jersey 08544

E-mail address: sahi@math.princeton.edu 